\title{
Shape Analysis of Consecutive Beats May Help in the Automated Detection of Atrial Fibrillation
}

\author{
Filip Plesinger ${ }^{1}$, Petr Andrla ${ }^{1}$, Ivo Viscor $^{1}$, Josef Halamek ${ }^{1}$, Veronika Bulkova ${ }^{2}$ and Pavel Jurak ${ }^{1}$ \\ ${ }^{1}$ The Czech Academy of Sciences, Institute of Scientific Instruments, Brno, Czech Republic \\ ${ }^{2}$ Medical Data Transfer, Brno, Czech Republic
}

\begin{abstract}
Background: Atrial fibrillation (AF) is associated with a higher risk of heart failure or death. AF may be episodic and patients with suspected $A F$ are equipped with Holter ECG devices for several days. However, automated detection of $A F$ in an ECG signal remains problematic, as was shown by the results of the PhysioNet Challenge 2017. Here, we introduce a simple yet robust logistic regression model for $A F$ detection.

Method: The detrended signal is filtered (1-35 Hz) and normalized. QRS detection based on envelograms (10-35 $\mathrm{Hz})$ reveals QRS complexes. Five features are extracted from the ECG signal describing RR stability as well as the shape stability of areas preceding QRS complexes. Features were extracted for 1,517 recordings from the PhysioNet Challenge 2017 public dataset (758 AF recordings and 759 recordings with normal rhythm, other arrhythmia or noisy signal). The recordings were split in a 70/30\% ratio for the purposes of training and testing.

Results: The results showed a sensitivity and specificity of $93 \%$ and $90 \%$, respectively (AUC 0.96). The presented model was also tested on the MIT-AFDB public database, showing sensitivity and specificity of 89 $\%$ and $88 \%$, respectively. However, tests on an independent private dataset revealed lower specificity when pathologies which are not widely present in the training dataset are common in the tested ECG signal.
\end{abstract}

\section{Introduction}

Atrial fibrillation (AF) is a disease resulting in chaotic and repeated activation of heart atriums. It is present in 1$2 \%$ of the general population [1]. Although atrial fibrillation itself is not life-threatening, it is associated with a prevalence of heart diseases such as hypertension and coronary artery diseases [2], stroke and also a higher morbidity of stroke events in AF patients [3].

Since AF may be episodic, patients suspected of this disease are fitted with a Holter ECG device and their ECG is acquired continuously for several days. However, this produces a large amount of data, for which reason automated methods of AF detection are needed. The search for automated algorithms for AF detection during the PhysioNet Challenge 2017 [4] showed many promising methods, usually focused on machine learning approaches such as random forests [5], recurrent and convolutional neural networks [6], [7], supported vector machines [8] and others. However, these robust methods usually use tens or hundreds of features and may be difficult to implement in miniature processing units. Therefore, we designed an extremely simplistic model based on logistic regression; it implements only five input features and should be easy to implement in hand-held devices.

\section{Method}

The presented method uses a 1-lead ECG signal with a sampling frequency of at least $250 \mathrm{~Hz}$. It was designed to analyse short ECG blocks of a length of up to 60 seconds.

First, the signal is detrended. Next, several transformed signals are prepared: BPN (band pass, 1-35 Hz, normalized), LFE (low-frequency envelogram, 1-8 Hz) and MFE (mid-frequency envelogram, 10-30 Hz).

Next, QRS complexes are detected using the MFE signal: peaks higher than the $85^{\text {th }}$ percentile and with a minimum distance of $0.25 \mathrm{~s}$ from each other are selected as QRS. The RR vector is computed as the difference of consecutive QRS locations.

\subsection{Feature extraction}

The presented approach implements five features. The first feature is the median RR interval (medRR).

The second feature describes the shape stability of the area preceding each QRS complex (apQRS). While the end of apQRS is fixed (74 ms before QRS), the width of apQRS is modified by RR intervals and is computed as follows:

$$
w_{\text {apQRS }}=0.135 \times \operatorname{medRR}[s]
$$


Then, the Pearson correlation is computed between apQRS regions of each two consecutive QRS complexes using BPN. Constants defining apQRS boundaries were optimized using brute force, as shown in Fig. 1. In total, we obtain $\mathrm{N}-1$ correlation values if $\mathrm{N}$ is the QRS count. Finally, the second feature is computed as the mean correlation value.

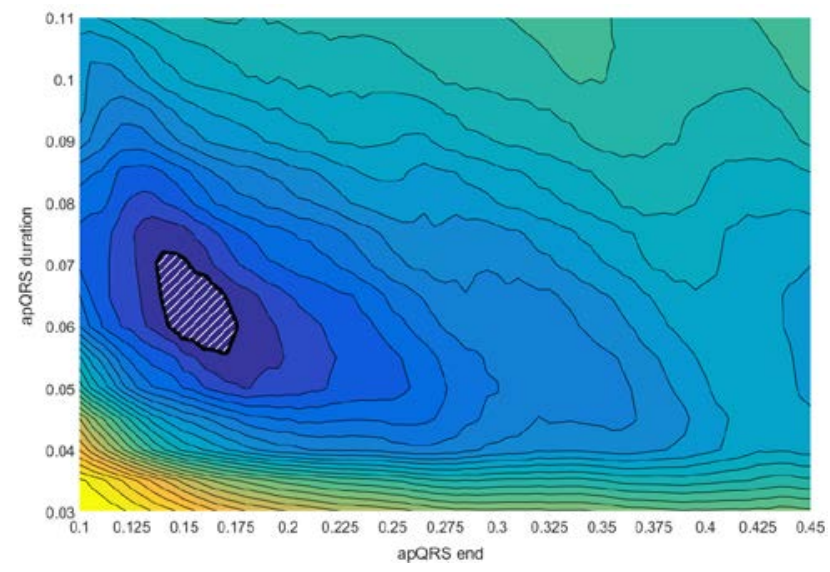

Figure 1. Optimization of apQRS boundaries. The hatched area identifies minimal error.

The third feature also describes the shape stability of the apQRS area, though separately for each beat. The standard deviation of apQRS (in the BPN signal) is measured for each QRS. The third feature is the mean of these standard deviations.

The fourth and fifth features describe RR stability and are based on the first derivative of RR intervals dRR. Therefore, the fourth feature is computed as the mean absolute value of dRR. Finally, the fifth feature is computed as the standard deviation of dRR.

\subsection{Model training}

We extracted these 5 features for 1,517 files from the PhysioNet Challenge 2017 [4]. Although it originally consisted of 8,138 files, only 758 were labelled as atrial fibrillation (AF). We used all AF recordings and added another 759 recordings labelled as Normal, Other Arrhythmia or Noisy Recording. We used the labelling provided by the Challenge. Next, we trained a logistic regression model. We used the Matlab ${ }^{\circledR}$ Classification Learner application from the Machine Learning and Statistics Toolbox. The dataset was divided in a 70/30 ratio into training and testing datasets.

\section{Results}

The logistic regression model (Tab. 1) showed a sensitivity and specificity of $93 \%$ and $90 \%$. The Area Under Curve (AUC) of the presented model was 0.96.

Table 1. Resultant logistic regression model. All feature estimates have a p-value $<0.0001$.

\begin{tabular}{llll}
\hline$\#$ & Feature & Estimate & \\
\hline- & (Intercept) & 6.2372 \\
1 & Median RR & -4.9030 \\
2 & Mean $\quad$ corr. & -5.2453 \\
& (apQRS) & \\
3 & Mean std (apQRS) & -77.6518 \\
4 & Mean abs (dRR) & 38.1098 \\
5 & Std (dRR) & -22.7520 \\
\hline
\end{tabular}

\subsection{Results from independent data}

In addition, we also tested the presented model on independent data. The model was tested using the public MIT-AFDB database [9]. A total of 605 ECG regions from 23 recordings were analysed. The sensitivity and specificity of detecting atrial fibrillation were $89 \%$ and $87 \%$, respectively.

The model was also tested on a private dataset (MDT, Czechia). This dataset $(250 \mathrm{~Hz})$ was divided into 8 classes (atrial fibrillation, AV block, noisy, premature atrial contractions, premature ventricular contractions, supraventricular tachycardia and ventricular tachycardia).

The results prepared using this dataset $(3,754$ recordings) showed a sensitivity and specificity of $90 \%$ and $66 \%$, respectively. This significantly reduced specificity is further discussed in the section Discussion.

\subsection{Implementation}

The presented model has also been implemented as an interactive plugin for the free SignalPlant software (Fig. 2) [10]. It uses multi-thread processing and, when the process is finished, shows the probability of atrial fibrillation during the whole recording (Fig. 2C - hatched areas). By default, processing is performed in 20 sec. regions with a 10 sec. step; 6 consecutive regions with $\mathrm{AF}$ probability $>0.5$ switch on AF event, and the AF event is switched off in the same manner. All presented constants are adjustable. After processing, the user may measure AF probability and BPM using the pointer; clicking on a specific point also changes the displayed time range in the main SignalPlant window. This is useful for examining specific locations when atrial fibrillation begins or ends. Finally, the plugin may export marks pointing to AF episodes. 


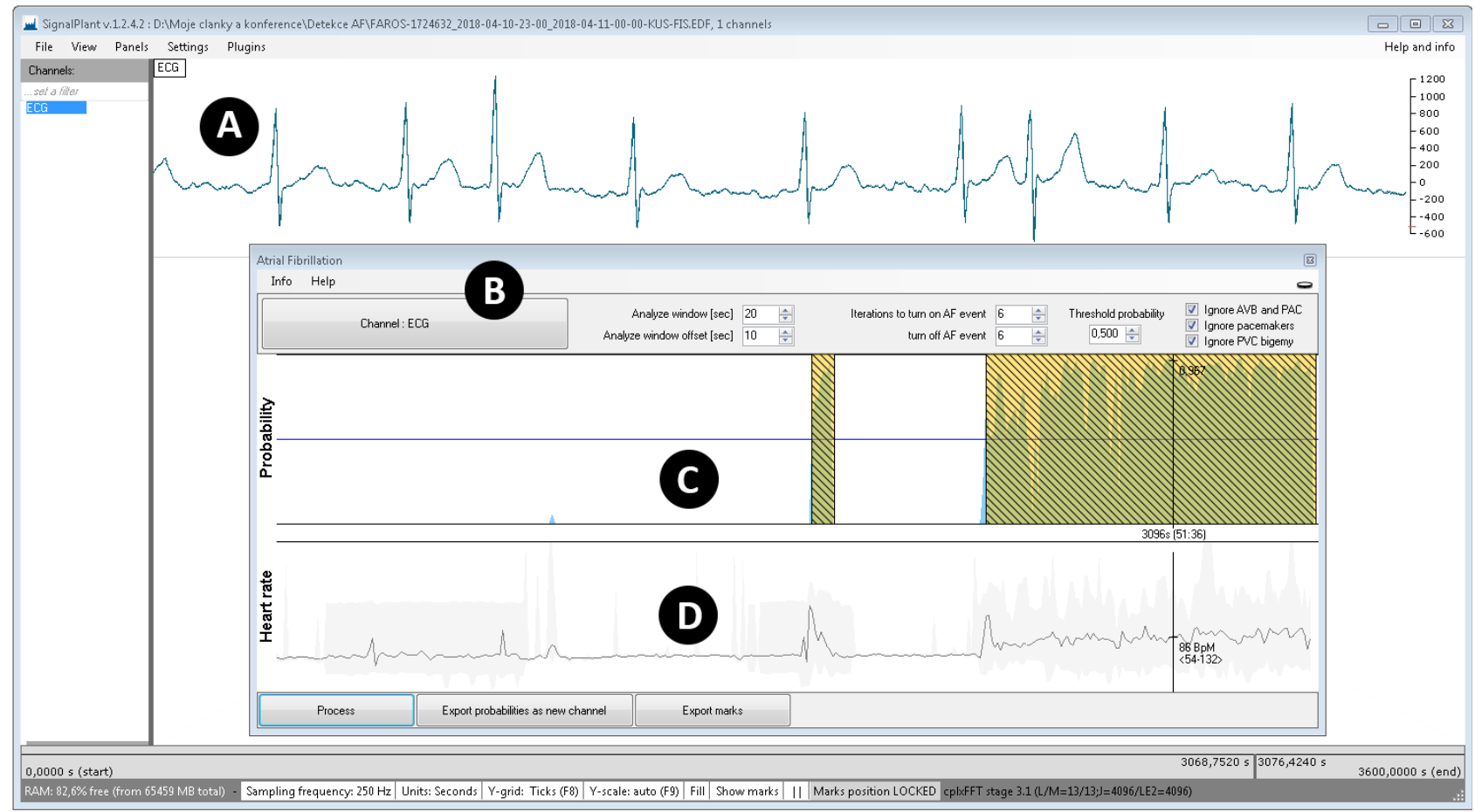

Figure 2. Software implementation of the presented model as a plugin for free SignalPlant [10] software (A). 1-hour ECG was loaded into SignalPlant; only 8 seconds of the signal is displayed. After processing, the plugin window (B) shows two panels: probability in time (C) and heart rate (D). The probability panel shows episodes of atrial fibrillation during the whole recording as hatched areas. The blue line is a threshold set at 0.5 by default; it can be changed. The heart rate panel (D) shows related BPM (median - black line, min/max - grey background). A click with the pointer in area (D) will show BPM values at a specific time and will also change the window displayed in (A); this allows the user to see the studied signal in detail.

\section{$4 \quad$ Discussion}

The results obtained using two public databases showed that the presented model should be a simple yet robust solution for the detection of atrial fibrillation.

Table 2: Output of the presented model for specific pathologies in the private dataset. The output is presented as the median probability of the atrial fibrillation class.

\begin{tabular}{lcc}
\hline Class & $\mathrm{N}$ & Probability \\
\hline Atrial fibrillation & 510 & 0.89 \\
AV block & 500 & 0.98 \\
Noisy & 366 & 0.18 \\
Premature atr. contr. & 446 & 0.06 \\
Premature vent. cont. & 449 & 0.13 \\
Sinus rhythm & 382 & 0.03 \\
Supr. vent. tachycardia & 508 & 0.45 \\
Vent. tachycardia & 593 & 0.09 \\
\hline
\end{tabular}

However, further examination on a private dataset (the company MDT, Czechia) showed similar sensitivity, but significantly lowered specificity. This means that some pathologies are likely to be misidentified as atrial fibrillation. Table 2 shows results for pathologies present in the private dataset. It is clear that the presented model reacts strongly to AV block recordings. Another issue may also follow from supra-ventricular tachycardia.

\subsection{Cases of false positivity}

The reason why the presented model reacts so strongly to AV block may be as follows: two of the five model features focus on the stability of blocks preceding QRS complexes (apQRS). Since this works perfectly for atrial fibrillation where these areas are not stable, the same applies in the case of $3^{\text {rd }}$ degree AV block where the Pwaves and QRS complexes run independently. The explanation as to why specificity was not as low during training and testing on the PhysioNet Challenge 2017 dataset may be a matter of balance: the Challenge dataset 
contains recordings from the general population, and since AV block is rare in the general population, it was also rare in the training dataset.

False positivity related to AV block may be corrected by thresholding. Preliminary tests on the private dataset showed that the thresholding of the standard deviation of RR intervals and the mean correlation of apQRS may decrease median probability for $\mathrm{AB}$ blocks from 0.98 to 0.0 . Moreover, this works with almost no negative effect on sensitivity to atrial fibrillation (the median probability for the AF class remains the same).

During our search for method weaknesses we also found that recordings with a pacemaker may lead to false positivity. This is also probably due to the fact that in such cases the apQRS area may contain only noise, and this noise will not correlate in consecutive beats (Tab. 1, feature \#2), leading to false reporting of atrial fibrillation. Therefore, some method (probably using higherfrequency ECG components) to detect pacing should be implemented to avoid false reporting of atrial fibrillation in paced beats.

Finally, we also found that ECGs containing premature ventricle contractions (PVC) in bigeminy linking may lead to false positive reporting of atrial fibrillation. This is caused by high standard deviation of RR intervals which is also common for atrial fibrillation. This might be corrected using RR histogram analysis, but the low number of beats may present a limit to this method. Therefore, we experimented with analysis of different envelograms near the QRS (KES, [11]) and preliminary results have shown that if KES values correlate highly with RR intervals, it is very likely PVC in bigeminy linking.

\section{Conclusion}

We designed a logistic regression model detecting atrial fibrillation in 1-lead ECG signals. Since this model implements only 5 features, it is easy to implement in hand-held devices. Although the results on the training/testing dataset (PhysioNet Challenge 2017), and also on an independent public database (MIT-AFDB), have shown strong sensitivity and specificity, further checks on a private dataset revealed strong false positivity in pathologies which are not so common in the training dataset. We have suggested ways of covering these observations; this also demonstrated possible weaknesses when using unbalanced datasets. The presented model has been implemented as a plugin for the free signal processing software SignalPlant.

\section{Acknowledgements}

This research was supported by project MSM 100651602 by the Czech Academy of Sciences and by project LO1212 by MEYS CR.

\section{References}

[1] G. Y. H. Lip et al., “Atrial Fibrillation,” Nat. Rev. Dis. Prim., vol. 2, p. 16016, Mar. 2016.

[2] G. V Naccarelli, H. Varker, J. Lin, K. L. Schulman, and A. F. L. Patients, "Increasing Prevalence of Atrial Fibrillation and Flutter in the United States," AJC, vol. 104, no. 11, pp. 1534-1539, 2009.

[3] A. J. Camm et al., "Guidelines for the Management of Atrial Fibrillation,” Eur. Heart J., vol. 31, no. July, pp. 2369-2429, 2010.

[4] G. D. Clifford et al., "AF Classification from a Short Single Lead ECG Recording: The Physionet Computing in Cardiology Challenge 2017," in Comput Cardiol (Rennes IEEE), 2017, pp. 1-4.

[5] C. Hoog Antink, S. Leonhardt, and M. Walter, "Fusing QRS Detection, Waveform Features, and Robust Interval Estimation with a Random Forest to Classify Atrial Fibrillation,” in Comput Cardiol (Rennes IEEE), 2017, vol. 44, pp. 1-4.

[6] M. Vollmer, P. Sodmann, L. Caanitz, N. Nath, and L. Kaderali, "Can Supervised Learning Be Used to Classify Cardiac Rhythms? Convolutional Neural Network,” in Comput Cardiol (Rennes IEEE), 2017, vol. 44, pp. 2-5.

[7] T. Teijeiro, C. A. García, D. Castro, and P. Félix, "Arrhythmia Classification from the Abductive Interpretation of Short Single-Lead ECG Records," in Comput Cardiol (Rennes IEEE), 2017, vol. 44, pp. 1-4.

[8] R. Smíšek et al., "SVM Based ECG Classification Using Rhythm and Morphology Features , Cluster Analysis and Multilevel Noise Estimation," in Comput Cardiol (Rennes IEEE), 2017, vol. 44, pp. 1-4.

[9] G. B. Moody and R. G. Mark, "A New Method for Detecting Atrial Fibrillation Using RR Intervals," Computers in Cardiology. pp. 227-230, 1983.

[10] F. Plesinger, J. Jurco, J. Halamek, and P. Jurak, "SignalPlant: an Open Signal Processing Software Platform,” Physiol. Meas., vol. 37, no. 7, pp. N38-N48, 2016.

[11] F. Plesinger, P. Klimes, J. Halamek, and P. Jurak, "Taming of the Monitors: Reducing False Alarms in Intensive Care Units,” Physiol. Meas., vol. 37, no. 8, pp. 1313-1325, 2016.

Address for correspondence:

Filip Plesinger

Institute of Scientific Instruments of the CAS, v.v.i.

Kralovopolska 147,

Brno 612 64, Czech Republic

E-mail address: fplesinger@isibrno.cz 